Bull. Mater. Sci., Vol. 7, No. 5, December 1985, pp. 491-497. (C) Printed in India.

\title{
Pressure dependence of electrical resistivities of some charge transfer complexes
}

\begin{abstract}
A T OZA
Department of Physics, Sardar Patel University, Vallabh Vidyanagar 388 120, India.

Abstract. The electrical resistivities of organic charge transfer complexes namely dithionaphthalene-iodine $(1: 1)$, anthracene-trinitrobenzene $(1: 1)$, pyrene-2 $I_{2}$ and benzidineTCNQ (dichloromethane) have been studied upto 2-8 GPa pressure. An increase in the electrical resistivities shows that the conduction is due to a hopping mechanism involving localized levels near the band edges or is trap-limited. Pressure can increase both the density of defects and intermolecular orbital overlap as opposite effects
\end{abstract}

Keywords. Charge transfer complexes; electrical resistivities; hopping conduction; trap levels; density of defects.

\section{Introduction}

Recently an increase in the electrical resistivities of some inclusion compounds of iodine at high pressures has been reported (Oza 1980, 1982, 1983, 1984). The increase in the electrical resistances of the samples was attributed to either the localization near the band edges (Oza 1982, 1984) or localization associated with the traps (Oza 1983). In the present work the pressure dependence of the electrical resistivities of four charge transfer complexes called dithionaphthalene-iodine $(1: 1)$, anthracene-trinitrobenzene $(1: 1)$, pyrene-2 $\mathrm{I}_{2}$ and benzidine-TCNQ (dichloromethane), where TCNQ $=7,7,8,8$ tetracyano-p-quinodimethane, has been reported to have similar nature which shows that the suggested conduction mechanism is not limited only to the inclusion compounds of iodine.

\section{Experimental}

Dithionaphthalene (DTN) was prepared by a direct reaction between sulphur and naphthalene in dimethylformamide medium as described in details elsewhere (Oza 1980). The chocolate-brown coloured dithionaphthalene and iodine were sublimated in a cosublimation growth tube to interact with each other in the vapour phase and the crystalline complex was collected on a glass rod as substrate. Blue needles of dimensions $3.0 \times 1.0 \times 0.1 \mathrm{~mm}^{3}$ having a metallic sheen were collected from the deposited complex and washed in toluene for removing unreacted iodine. Anthracene-TNB (1:1) ( TNB $=$ trinitro-benzene) was prepared from a mixed solution of anthracene and trinitrobenzene as per Foster (1969). The scarlet coloured complex was crystallized with dimensions $4.0 \times 2.0 \times 0.1 \mathrm{~mm}^{3}$ using an evaporation technique. This anthracene-TNB complex is reported to have high mobility. Pyrene- $2 \mathrm{I}_{2}$ the blue semiconducting complex was prepared by evaporating the mixed solution according to the method discussed by 
Kommandeur and Hall (1961). It is a well-known organic semiconductor (Singer and Kommandeur 1961).

The solvent-included clathrate benzidine-TCNQ (dichloromethane) was prepared by mixing hot solutions of benzidine and TCNQ in dichloromethane $\left(\mathrm{CH}_{2} \mathrm{Cl}_{2}\right)$ in equimolar proportion. The solvent molecules are found to be accommodated in the channels between the stacks in this complex (Ikemoto et al 1972; Kuroda 1976).

The electrical resistivities upto $8 \mathrm{GPa}$ pressure were determined using a Bridgmann anvil apparatus, constant current source and a d.c. null detector. The sample-electrode contacts were found to be non-rectifying and the contact resistances were much lower than the high resistances of the single crystals of the complexes. Various tests failed to show ionic conduction.

\section{Results}

The room temperature resistivity of the chocolate-brown dithionaphthalene single crystals along the long direction of growth was found to be $p=10^{6}-10^{7} \mathrm{ohm}-\mathrm{cm}$. The resistivity at $1000 \mathrm{~Hz}$ was $\rho_{\text {a.c. }}=10^{4} \mathrm{ohm}-\mathrm{cm}$. Thus the electrical resistivity of the donor itself was in the semiconducting range. The room temperature resistivity in the needle direction of the crystals of DTN-I $2(1: 1)$ complex was $4.8 \times 10^{5} \mathrm{ohm}-\mathrm{cm}$. This resistivity dropped by two or three orders of magnitude. Anthracene-TNB $(1: 1)$ crystals had d.c. resistivity of about $2.0 \times 10^{8} \mathrm{ohm}-\mathrm{cm}$ and a.c. resistivity (at $1000 \mathrm{~Hz}$ ) of about $10^{6} \mathrm{ohm}-$ $\mathrm{cm}$. The crystals were cut and mounted on the anvil so as to measure the electrical resistances along this direction. The electrical resistivities of pyrene-2 $\mathrm{I}_{2}$ crystals lie in the range of 10-50 ohm-cm (Oza 1980; Kommandeur and Hall 1961). At $1000 \mathrm{~Hz}$ the resistivity dropped by two or three orders of magnitude. The benzidine-TCNQ $\left(\mathrm{CH}_{2} \mathrm{Cl}_{2}\right)$ inclusion complex indicated electrical resistivities of the order of $10^{6} \mathrm{ohm}-\mathrm{cm}$ in the form of polycrystalline pellets prepared at $2.5 \times 10^{6} \mathrm{~Pa}$ of pressure. This complex was studied at high pressure in the form of pellet only.

An increase in resistivities at high pressure is found for all the materials (figures 1-5). The electrical resistivity increased from $1.2 \times 10^{6} \mathrm{ohm}-\mathrm{cm}$ to $2.0 \times 10^{6} \mathrm{ohm}-\mathrm{cm}$ for dithionaphthalene crystal when the pressure was increased from the atmospheric pressure to $1.8 \mathrm{GPa}$. There is complete round-off in the resistivity vs pressure curve in

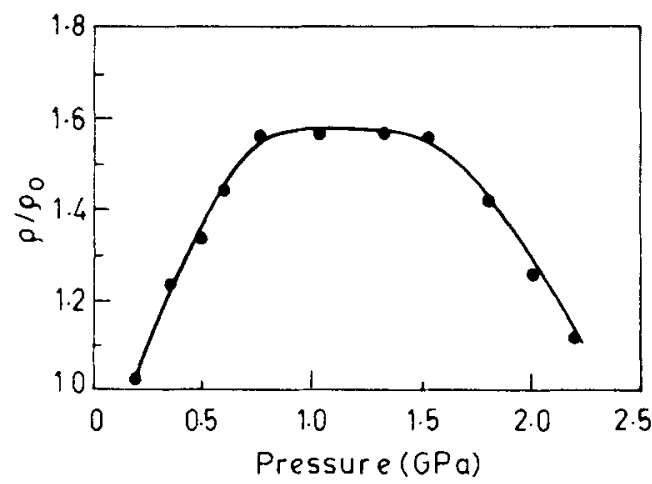

Figure 1. Pressure dependence of electrical resistivity of dithionaphthalene. 


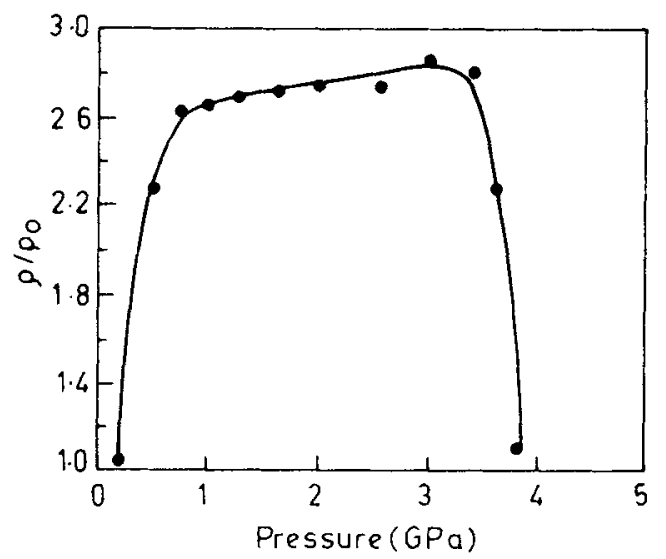

Figure 2. Pressure dependence of electrical resistivity of dithionaphthalene-iodine $(1: 1)$.

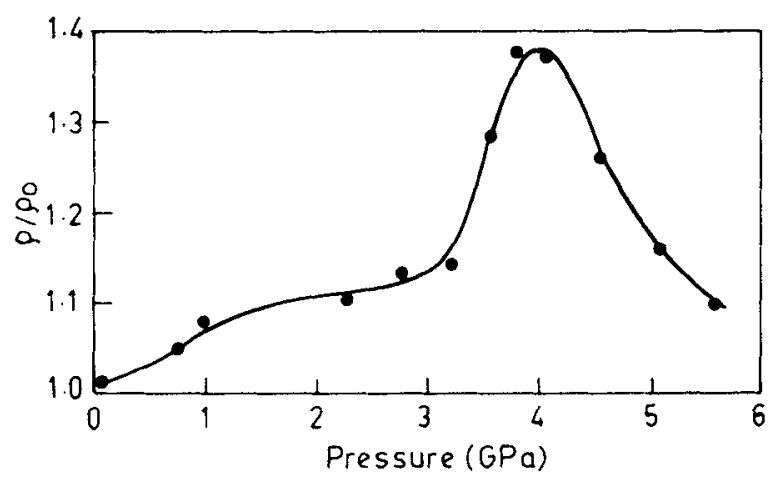

Figure 3. Pressure dependence of electrical resistivity of anthracene-trinitrobenzene $(1: 1)$.

this pressure range (figure 1). The $\mathrm{DTN}_{2}(1: 1)$ crystals showed similar behaviour (figure 2). In both the materials the resistivity dropped after a range of saturation The crystals of anthracene-TNB $(1: 1)$ and pyrene- $2 \mathrm{I}_{2}$ complexes exhibited slightly different modes of increase in the electrical resistivities at high pressure (figures 3,4 ). A resistivity peak around $4 \mathrm{GPa}$ was found for anthracene-TNB and around $2 \mathrm{GPa}$ for pyrene-2 $\mathrm{I}_{2}$. The resistivity increased at lower pressures in the case of anthracene-TNB while it dropped in pyrene- $2 \mathrm{I}_{2}$. The resistivity vs pressure curve for benzidine-TCNQ $\left(\mathrm{CH}_{2} \mathrm{Cl}_{2}\right)$ pellet is a complete round-off like amylose-iodine (Oza 1983) with the maximum increase in the resistivity of one order of magnitude (figure 5). The increase in the resistivities of about $6-12$ for benzidine-TCNQ $\left(\mathrm{CH}_{2} \mathrm{Cl}_{2}\right)$ and pyrene-2I $\mathrm{I}_{2}$ were found to be comparatively larger than that of anthracene-TNB and $\mathrm{DTN}_{2}$.

\section{Discussion}

There are two possibilities regarding the conduction mechanism in these complexes. Either there is thermally-activated hopping near the band edges or there is trap-limited 


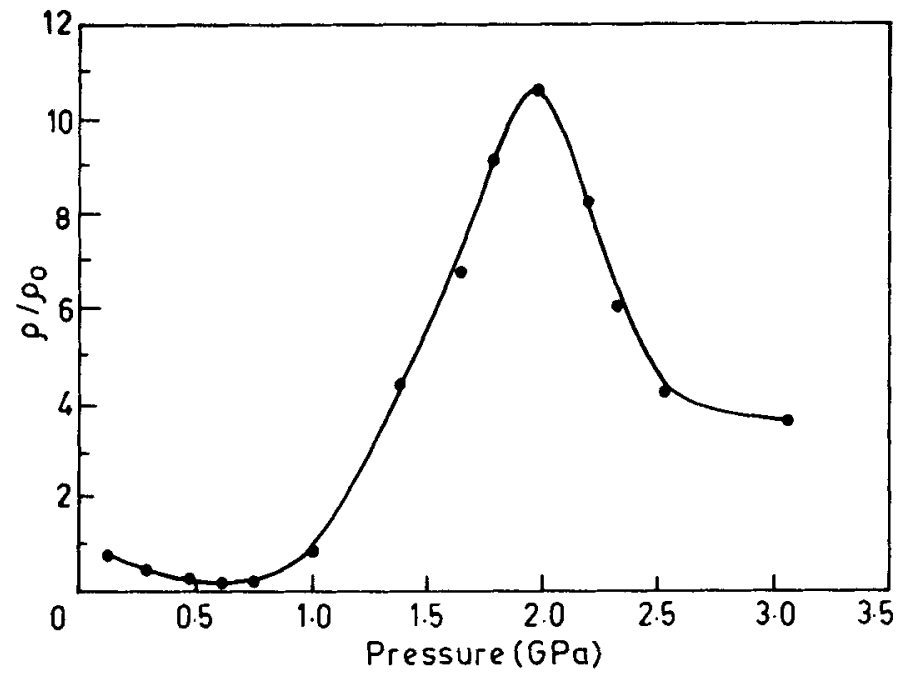

Figure 4. Pressure dependence of electrical resistivity of pyrene-2 $\mathbf{I}_{2}$.

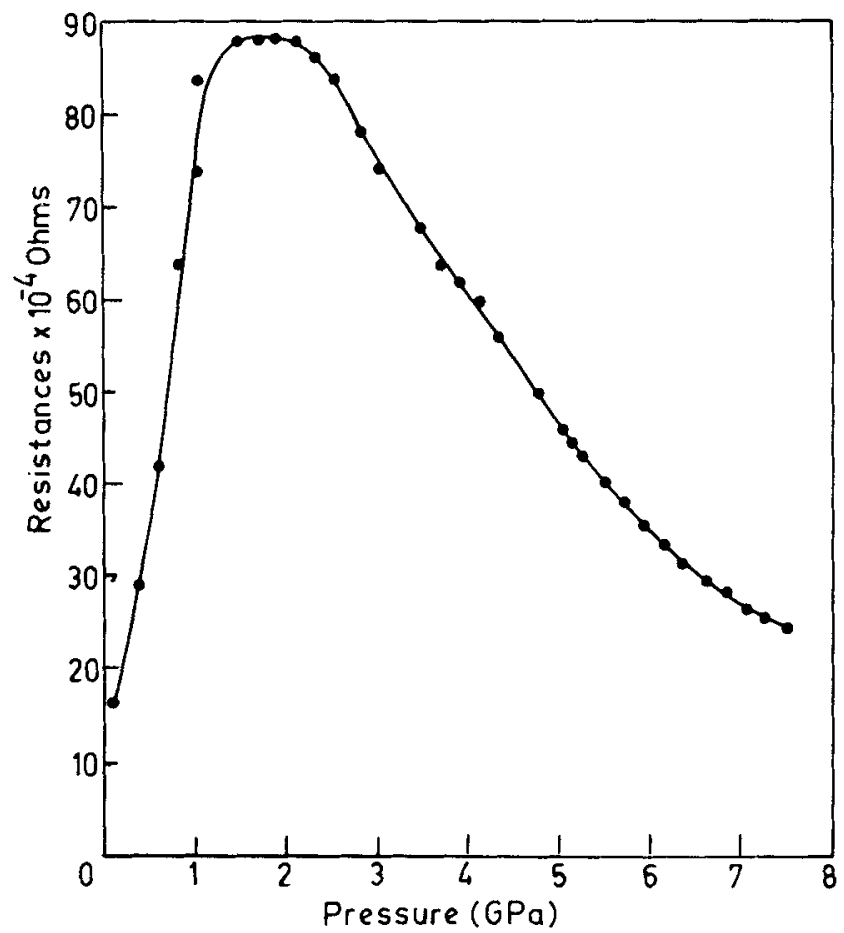

Figure 5. Pressure dependence of electrical resistivity of benzidine-TCNQ (dichloromethane).

conduction. Both the models can explain the increase in the resistivities at high pressures and the Arrhenius-type temperature dependence of the resistivities of the charge transfer complexes studied in the present work, and the inclusion complexes of iodine (Oza 1982, 1983). In the first mechanism of conduction it is possible that the charge 
carriers are excited across a large band gap preceded and followed by the hopping motions among the localized states near the band edges, i.e. far away from the Fermi level (Mott 1969). On the other hand, the trap-limited mechanism explains the saturation of electrical resistances of the samples at high pressure as traps are limited in number and excess electrons then contribute to conduction. The saturation of electrical resistances at high pressures also reveals that the depths of the traps rather than the density of the traps increase more effectively under pressure. A transition in the trapping probability when a large group of traps crosses the Fermi level is a consequence of the change in the depth of the traps and contributes to the saturation of electrical resistivities at high pressure (Oza 1983). The saturation ranges of DTN and DTN I crystals in the present work can be ascribed to these factors while the decrease in the resistivities of all the materials in the high pressure range can be attributed to the averaging over the specific directions due to increased dimensionality of electronic transport at high pressures.

Organic donors are generally electrical insulators. However, some like tetrathiotetracene (TTT), violanthrene, etc. are ele trical conductors while pyrene and others conduct at high pressures (Perez-Albuerne et al 1971; Drickamer 1965). DTN is semiconducting like TTT. The electrical conduction in these donors with sulphurbridges seems to be mainly due to the proper columnar stacking of the molecules with small stacking distances arising out of the attractive dipole-dipole interaction between neighbouring molecules with large dipole moments. There are two C-S bonds in each molecule of DTN; a C-S bond has a large dipole moment of about 3.0 Debye which increases molecular polarizability (Ferguson 1973). The increase in the electrical resisti ity of DTNat high pressure shows the disordered nature of these molecular stacks.

The similarity between the resistivity vs pressure curves for DTN and DTN-I ${ }_{2}$ shows that the major conduction remains along the DTN stacks in the DTN- $\mathrm{I}_{2}$ complex. Iodine increases the density of free charge carriers in the conduction band and therefore drops the electrical resistivity of DTN in DTN- $\mathrm{I}_{2}$ as it usually oocurs in charge transfer complexes (Perlstein 1977), but it also increases the density of trap-levels due to the disordered nature of iodine chains which is usually found in iodine complexes (Saenger 1975). Because of this, the resistances of $\mathrm{DTN}-\mathrm{I}_{2}$ samples increases further at high pressures. All the trap levels are filled and saturation occurs only when many charge carriers are trapped.

On the other hand in anthracene-TNB, traps most probably lie deep in the forbidden gap since it requires high pressure to increase the resistivities significantly. This is consistent with the large band gap. The high mobility of anthracene-TNB found in an earlier study reveals that the conduction is not due to the excitation of charge carriers across a well-defined gap but is some type of hopping conduction or is governed by traps.

Pyrene-2 $\mathrm{I}_{2}$ is a highly conducting compound in the present series and is a well-known semiconductor with a small band gap of $0.1 \mathrm{eV}$ (Singer and Kommadeur 1961). It is also clear that pyrene stacks conduct and iodine simply removes a partial charge from each pyrene molecule. The electrical resistivity of pyrene- $2 \mathrm{I}_{2}$ is found to drop at initial pressure, this may be related to the fact that the electrical resistivity of pyrene is found to drop at high pressure (Drickamer 1965). A comparison of the data of DTN and DTN $\mathrm{I}_{2}$ with these results implies that iodine only modifies the disorders along the DTN stacks or increases the density of traps while in pyrene- $2 \mathrm{I}_{2}$ the conduction is limited by the traps introduced by iodine only. Finally, there is a large increase of about one order of 
magnitude in the resistivity of pyrene- $2 \mathrm{I}_{2}$ under pressure which is most probably the result of additional localization near the band edges separated by a small forbidden energy gap. The increase in the density or depth of traps is limited by the small band gap.

In the benzidine-TCNQ $\left(\mathrm{CH}_{2} \mathrm{Cl}_{2}\right)$ inclusion complex, the major conduction is along the benzidine stacks and the environment of these stacks is disordered in nature because of the weakly bonded disordered sub-lattice of the solvent molecules. The resistivity vs pressure curve on the polycrystalline pellet studied here is very similar to that of amylose-iodine (Oza 1983). The arrhenius-type temperature dependence at three clamped pressures clearly discards the possibility of any change in the density of traps by the applied pressure (A T Oza, A K Bandyopadhyay and S V Subramanyam, unpublished results). Thus pressure enhances the effect of trapping only by increasing the depths of the traps. The electronic wavefunctions sharply cut off for deep-lying traps and therefore, the degree of localization of a trap depends on its depth from the band edges.

The electrical resistivity of several charge transfer complexes increases with pressure for $p \leqslant 2$ Gpa and decreases at higher pressure. Such a behaviour can be also a consequence of two opposite effects. Pressure increases both the density of defects and the intermolecular coupling (Drickamer 1965). The increase in defects can be due to generation of defects by plastic deformation (Schnaithamann and Wolf 1965). The electrical resistivity increases due to increase in the density of defects at high pressure but this is overcome by a decrease in the resistivity because of the strengthening of intermolecular coupling at still higher pressures.

The present work also reveals that the observed pressure dependence is not only limited to the inclusion compounds of iodine but is also a characteristic of anthraceneTNB, benzidine-TCNQ $\left(\mathrm{CH}_{2} \mathrm{Cl}_{2}\right)$ and DTN itself in which the ionic mechanisms of conduction are less likely. It also proves that this pressure dependence of resistivity is independent of the interactions taking part in complex formation and is a characteristic of some of the disordered organic semiconductors both with and without significant charge transfer.

\section{Acknowledgements}

The author is thankful to the DST and the NCERT, New Delhi, for providing financial support. The author thanks Profs. M K Agarwal, A R Patel and M S Joshi for their kind co-operation.

\section{References}

Drickamer H G 1965 Solid state physics, Advances in research and applications (eds) F Seitz and D Turnbull (New York, London: Academic Press) vol. 17, p. 1 and references therein

Ferguson L N 1973 The modern structural theory of organic chemistry (New Delhi: Prentice Hall of India) p. 200.

Foster R 1969 Organic charge transfer complexes, (London, New York: Academic Press) and references therein

Ikemoto I. Chikaishi K. Yakushi K and Kuroda H 1972 Acta Cryst. B28 3502

Kommandeur J R and Hall F R 1961 J. Chem. Phys. 34129

Kuroda H 1976 Acta Cryst. B28 3502 
Mott N F 1969 Philos. Mag. 19835

Oza A T 1980 Electrical conductivity of some organic polyiodide chain complexes Ph.D. thesis, Indian Institute of Science, Bangalore, India

Oza A T 1982 Phys. Status Solidi B114 K171

Oza A T 1983 Phys. Status Solidi 80573

Oza A T 1984 Crystal Research and Technology 16697

Perez-Albuerne E A, Johnson H Jr and Trevoy D J 1971 J. Chem. Phys. 551547

Perlstein J H 1977 Angew. Chem. Int. Ed. Engl. 16519

Saenger W 1975 Proceedings of the 8th Jerusalem symposium environmental effects on molecular structure and properties, Jerusalem (ed) B Pullman (Dordecht-Boston: D Reidel) p. 265

Schnaithmann R and Wolf R C 1965 Z. Naturforschung A20 76

Singer L S and Kommandeur J R 1961 J. Chem. Phys. 34133 